\title{
Os Odontoideum in a Patient with Cervical Vertigo: A Case Report
}

\author{
Jacopo Galli, MD,* Tommaso Tartaglione, MD,+ Lea Calo, $M D,{ }^{*}$ and \\ Fabrizio Ottaviani, $M D^{*}$
}

\begin{abstract}
Cervical vertebral anomalies are often associated with malformations or traumas, they may be completely asymptomatic and represent an occasional finding in vertigo or can cause severe neurologic complications (ie, compression of the upper cervical spine with myelopathy, epilepsy, or respiratory failure). This clinical case is a patient who came to us for observation for a peripheral harmonic vestibular syndrome, and in whom a malformation of the cervical vertebral joint (os odontoideum) was occasionally found on magnetic resonance imaging.
\end{abstract}

(Am J Otolaryngol 2001;22:371-373. Copyright (C) 2001 by W.B. Saunders Company)

(Editorial Comment: This report shows that not all vertigo is attributed to the end-organ. In this patient, brainstem compression caused by a congenital abnormality caused vestibular symptomotology.)

The first 2 cervical vertebrae, $\mathrm{C} 1$ and $\mathrm{C} 2$, constitute the occipitoatlantoaxial joint closely correlated with the cervicomedullary region. Congenital iatrogenic malformations of the cervical vertebral joint or secondary to chronic inflammation can cause compression of nerve structures, with subsequent impairment of the medulla oblongata, brainstem, spinal marrow, nerve roots, and several symptoms according to the involved structures. ${ }^{1}$

We report on a clinical case of a patient with a malformation of the cervical vertebral joint with basilar invagination, os odontoideum consequent to truncoenchephalic deformation, occasionally observed after the onset of symptoms of peripheral cervical vertigo with no radiographic evidence of bone instability or clinical symptoms of paresthesia or correlated motor deficits. Possible disorders of the cervical vertebral joint, with correlated

From the Institutes of ${ }^{*}$ Otorhinolaryngology and $†$ Radiology, University of the Sacred Heart ROMA, Rome, Italy.

Address reprint requests to Jacopo Galli, Clinic Otorhinolaryngology, University of the Sacred Heart ROMA, Largo A. Gemelli N8 00168 Roma, Italy.

Copyright $\odot 2001$ by W.B. Saunders Company

0196-0709/01/2205-0013\$35.00/0

doi:10.1053/ajot.2001.26503 clinical manifestations and probable impact on the vestibular apparatus, were also considered.

\section{CASE REPORT}

A 48-year-old male patient came to our observation for the abrupt onset of symptoms characterized by objective rotary vertigo and grade 2 spontaneous nystagmus toward the right side with segmental tonic harmonic deviations without subjective hypoacusis or acousmas. An objective, ear, nose, and throat examination did not show any no significant alterations. The case history noted a cranial trauma at the age of 19 years and episodes of epilepsy at the age of 13 years, which were treated with phenobarbital ( 1 tablet per day).

Audiometric tests showed moderate bilateral neurosensory hypoacusis, more marked on the left side for acute frequencies; whereas acoustic impedance and truncocephalic auditory-evoked potentials were normal.

After 3 days of corticosteroid therapy (methylprednisolone $40 \mathrm{mg} /$ daily), the patient reported symptom release with modest residual sense of instability on walking. Vestibular control tests (spontaneous tests, evoked vestibular symptoms, Fitzgerald-Hallpike caloric bithermic stimulation, and dynamic posturography) documented the absence of nystagmus, bilateral normoreflexia, composite equilibrium score 79, and normal somatic and visuovestibular components on sensory analysis. Radiographs of the cervical spine showed 
abnormally increased cervical lordosis, diffuse spondylosis, synostosis of spinous apophyses C1 and C2, marked bilateral narrowing of third junction foramen, and presence of bilateral C7 cervical rib. Doppler ultrasound of epiaortic vessels showed normally oriented flow of vertebral arteries, dominant on the right.

Although subsequent cranial magnetic resonance imaging ruled out malignant lesions of the brain, pontocerebellar angle, internal auditory canals, it did detect the presence of a malformation of cervical vertebral junction with basilar invagination and odontoid process encroaching on the posterior cranial fossa (Fig 1). A computed tomography (CT) study of the cervical vertebral junction in flexion and extension confirmed the basilar invagination, with the dens axis extending into the foramen magnum and the assimilation of the atlas to the occipital bone and occipital condyles. The dens axis seemed displaced dorsally, $1.7 \mathrm{~cm}$ from the anterior atlantal arch and fragmented apex (os odontoideum). The sagittal diameter of bulbomedullary junction was approximately $1 \mathrm{~cm}$, with no significant changes in dynamic flexion and extension tests (Fig 2).

Clinically, the patient did not show relevant sensorimotor deficits, and no such alterations were observed on somatosensory and motorevoked potentials studies.

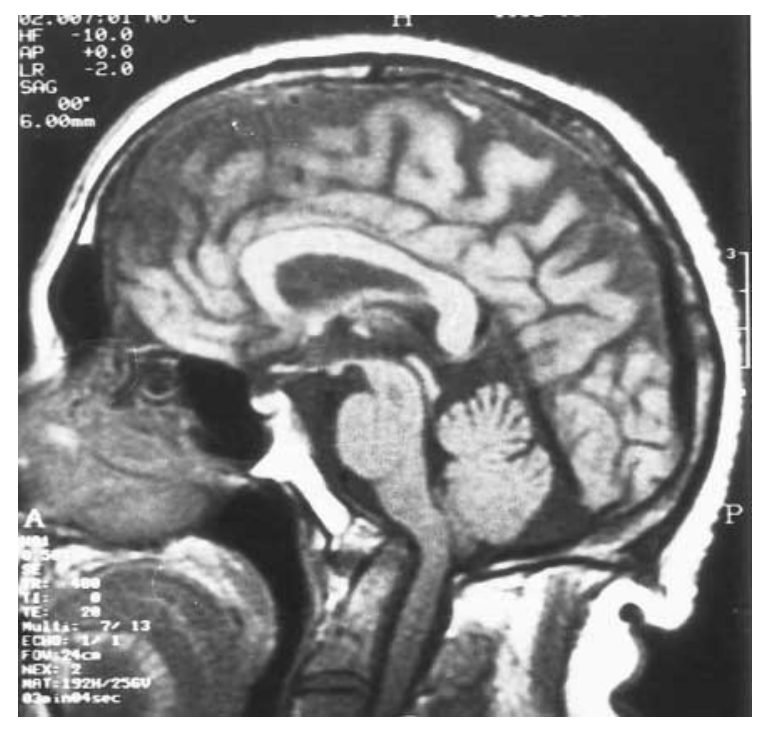

Fig 1. MRI detects the presence of a malformation of cervical vertebral junction with basilar invagination and odontoid process encroaching on the posterior cranial fossa.

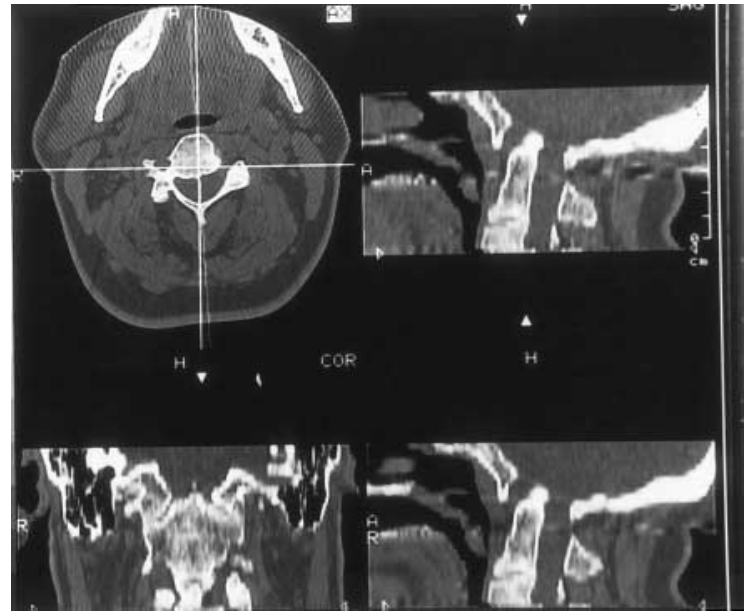

Fig 2. CT study of cervical vertebral junction in flexion and extension shows the basilar invagination with the dens axis extending into the foramen magnum and the assimilation of the atlas to the occipital bone and occipital condyles. The dens axis seemed displaced dorsally, $1.7 \mathrm{~cm}$ from the anterior atlantal arch and fragmented apex (os odontoideum). The sagittal diameter of bulbomedullary junction was approximately 1 $\mathrm{cm}$, with no significant changes in dynamic flexion and extension tests.

Considering the radiologic pattern, however, the consultant neurosurgeon advised transferring the patient to neurosurgery to plan an operation to stabilize the cervical tract by transmandibular route. Aware of the involved risk, the patient refused to undergo this treatment. At 1-year otolaryngologic and neurologic follow-up in the outpatient service, the patient's clinical situation was unchanged with normal somatosensory and motor potentials.

\section{DISCUSSION}

The reported case concerns a patient with acute peripheral vertigo associated with neurovegetative self-limiting disorders who underwent careful examination of the auditory function and neuroradiology. No alteration of the vestibular apparatus was detected; however there was a severe malformation of cervical vertebral junction.

Os odontoideum is a severe malformation of cervical vertebral junction with basilar invagination radiologically classified in 3 types: type 1 (round os odontoideum), where the epistrophic body is roundish at the base of odontoid process; type 2 (cone os odontoi- 
deum), where the epistrophic body is triangular at the base; and type 3 (blunt-tooth os odontoideum), where the epistrophic body is represented by a small process at the odontoid base. $^{2}$

From the time of its identification by Giacomini in $1886,{ }^{3}$ the question of a possible congenital origin for os odontoideum was debated, ${ }^{4}$ supported by some authors because of the association with syndromes as trisomy 21, Klippel-Feil syndrome, and multiple epiphyseal dysplasia. ${ }^{5}$ Furthermore, there is a posttraumatic acquired ${ }^{6}$ form correlated with the lack of fusion after fracture or secondary to vascular impairment consequent to the trauma with sclerosis and bony irregularities.

Symptoms reported by patients are complex and include cervical pain, torticollis, radiculopathy, nausea, vertigo, nystagmus, dysarthria, dysphonia, dysphagia, visual disorders, clinical signs of myelopathy, cervical and cerebral ischemia with epilepsy, syncope, and, in extremely severe cases, respiratory failure caused by damaged respiratory centers. The latter is mainly seen in severe congenital deformities or traumas. ${ }^{7-9}$

The observation of os odontoideum encroaching on the posterior cranial fossa, as we observed, is rare in the absence of neurologic symptoms associated with normal motor and somatosensory potentials and concomitant peripheral vertigo.

A cervical cause of vertigo is, however, of difficult assessment. ${ }^{10}$ The observation of a cervical nystagmus induced by cervical torsion was shown to be a normal cervicoocular reflex (COR). ${ }^{11}$ Reduced vestibular input or increased proprioceptive sensibility favor the appearance of COR; however, it alone does not establish the diagnosis of cervical vertigo. It can be suggestive of a cervical involvement in the referred symptomatology, and the diagnosis will be confirmed by its correlation with other pathologic cervical findings.

In our case, the increased cervical proprioceptive sensibility associated with the observed malformation might be a possible concomitant cause of symptoms; in the absence of a definite clinical demonstration, so as to agree with what is reported in literature.

Diagnostically, most cervical vertebral anomalies can be detected with a radiograph in laterolateral, posteroanterior, and oblique projections with the mouth open and in flexion and extension. The latter provide information on the motion and stability of cervical vertebrae. A better resolution of vertebral bodies is provided by CT with the 3-dimensional reconstruction of the images. MRI is complementary to CT for evaluating the possible involvement of brainstem structures, the basilar invagination caused by the extension of the dens of axis into the foramen magnum, the assimilation of the atlas with the occipital bone, and the consequently reduced sagittal diameter of bulbomedullary junction.

Considering the high risk of associated neurologic complications (eg, myelopathy, epilepsy, respiratory failure), an occasional finding of os odontoideum stresses the need, in cases of vestibular deficit, to complete the clinical study with diagnostic imaging (CT and MRI) for an exhaustive evaluation of cervical, vertebral, occipital, and brainstem structures.

\section{REFERENCES}

1. Rosenberg WS, Salame KS, Shumrick KV, et al: Compression of the upper cervical spine cord causing symptoms of brainstem compromise. Spine 13:14971500, 1998

2. Matsui H, Imada K, Tsuji H: Radiographic classification of os odontoideum and its clinical significance. Spine 15:1706-1709, 1997

3. Giacomini C: Sull'esistenza Dell'os-odontoideum Nell'uomo. Gior DR Acad di Med di Torino 65:26-38, 1886

4. Morgan MK, Onofrio BM, Bender CE: Familial os odontoideum. J Neurosurg 70:636-638, 1989

5. Manaligod JM, Bauman NM, Menezes AH, et al: Cervical vertebral anomalies in patients with anomalies of the head and neck. Ann Otol Rhinol Laryngol 108:925933, 19996

6. Verska JM, Anderson PA: Os odontoideum. A case report of one identical twin. Spine 22:706-709, 1997

7. Takakuwa T, Hiroi S, Hasegawa H, et al: Os odontoideum with vertebral artery occlusion. Spine 19:460462, 1994

8. Biesinger E: Vertigo caused by disorders of the cervical vertebral column. Diagnosis and treatment. Adv Otorhinolaryngol 39:44-51, 1988

9. Link DT, McCaffrey TV, Link M, et al: Cervicomedullary compression: An unrecognized cause of vocal cord paralysis in rheumatoid arthritis. Ann Otol Rhinol Laryngol 107:462-471, 1998

10. Brandt T: Cervical vertigo reality or fiction? Audiol Neurotol 4:187-196, 1996

11. Norrè ME: Cervical vertigo Diagnostic and semiological problem with special emphasis upon "cervical nystagmus." Acta Otorhinolaryngol Belg 41:436-450, 1987 